\title{
Diabetes Gestacional: Serão os Atuais Critérios de Diagnóstico Mais Vantajosos?
}

\section{Gestational Diabetes Mellitus: Is There an Advantage in Using the Current Diagnostic Criteria?}

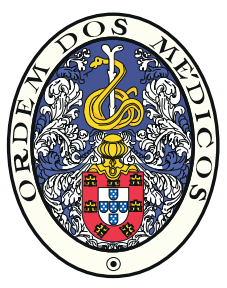

\author{
Ana Filipa FERREIRA $\otimes^{* 1,2}$, Catarina Miranda SILVA ${ }^{* 1,2}$, Dora ANTUNES ${ }^{1}$, Filipa SOUSA ${ }^{1}$, António Carlos LOBO ${ }^{1,2}$, \\ Paulo MOURA ${ }^{1,2}$ \\ Acta Med Port 2018 Jul-Aug;31(7-8):416-424 • https://doi.org/10.20344/amp.10135
}

\section{RESUMO}

Introdução: Não existe consenso internacional quanto aos critérios de diagnóstico da diabetes gestacional. Em Portugal, os critérios de Carpenter e Coustan foram substituídos por uma adaptação dos critérios da International Association of Diabetes and Pregnancy Study Groups. O objetivo deste estudo foi comparar a incidência e outcomes obstétricos/perinatais das grávidas com diabetes gestacional segundo os critérios atuais e prévios.

Material e Métodos: Estudo retrospetivo de 1218 gestações únicas complicadas com diabetes gestacional cuja vigilância/parto ocorreu entre 2008-2015. Consideraram-se dois grupos: diagnóstico pelos critérios da Direção Geral da Saúde - International Association of Diabetes and Pregnancy Study Groups (grupo 1); diagnóstico segundo Carpenter e Coustan (grupo 2), tendo sido feita análise estatística comparativa.

Resultados: A incidência da diabetes gestacional duplicou $(9,4 \%$ vs $4,6 \%)$ e o número de consultas/ano aumentou consideravelmente ( 3000 vs 2 000). No grupo 1 verificou-se um risco inferior de recém-nascidos macrossómicos em relação ao grupo 2 [RR 0,44 (IC (95\%): 0,26 - 0,76)], e um risco mais elevado de recém-nascidos leves para a idade gestacional (LIG) [RR 1,99 (IC (95\%):1,19-3,31)]; um risco cerca de seis e quatro vezes superior de hipoglicémia [RR 6,30 (IC (95\%): 3,39 - 11,71)] e hiperbilirrubinémia [RR 3,89 (IC (95\%): 2,25 - 6,72)] neonatais, respetivamente. Não houve diferenças em relação a outros outcomes.

Discussão: A redução dos recém-nascidos macrossómicos não resultou em melhoria dos outcomes, havendo um aumento dos recém-nascidos leves para a idade gestacional bem como de complicações neonatais. Os critérios atuais poderão associar-se a maiores gastos em saúde, devido ao aumento considerável da incidência de diabetes gestacional e maior vigilância em consultas, sem benefícios obstétricos/perinatais.

Conclusão: A aplicação dos critérios da Direção Geral da Saúde - International Association of Diabetes and Pregnancy Study Groups associou-se a redução da macrossomia, não acompanhada de uma melhoria dos outcomes. É discutível o benefício destes critérios em relação aos anteriormente preconizados.

Palavras-chave: Diabetes Gestacional/diagnóstico; Diabetes Gestacional/epidemiologia; Portugal

\section{ABSTRACT}

Introduction: There is no international consensus regarding gestational diabetes mellitus diagnostic criteria. In Portugal, the Carpenter and Coustan criteria were replaced by an adaptation of the International Association of Diabetes and Pregnancy Study Groups criteria. Our aim was to compare the incidence and outcomes of pregnancies complicated by gestational diabetes mellitus according to the current and previous criteria.

Material and Methods: Retrospective analysis of 1218 singleton pregnancies complicated with gestational diabetes mellitus, with surveillance/delivery between 2008-2015. Two groups were considered: identification according to the Directorate-General of Health criteria - International Association of Diabetes and Pregnancy Study Groups (group 1); identification through Carpenter and Coustan criteria (group 2). A comparative analysis was performed.

Results: The incidence of gestational diabetes mellitus doubled (9.4\% vs 4.6\%), and the number of consultations/year increased ( 3000 vs $\sim 2000)$. In Group 1, in comparison with group 2, there was a lower risk of macrosomia in newborns [RR 0.44 (IC (95\%):0.26 - 0.76)] and a higher risk of small for gestational age infants [RR 1.99 (IC (95\%):1.19 - 3.31)]; a 6 - fold and 4 fold higher risk in neonatal hypoglycemia [RR 6.30 (IC (95\%): 3.39 - 11.71)] and hyperbilirubinemia [RR 3.89 (IC (95\%): 2.25 - 6.72 )] were also observed, respectively. There were no differences regarding other outcomes.

Discussion: Outcomes related to the decrease in macrosomia did now show any improvement, with even an increase in Small for Gestational Age and neonatal complications. Given the increased incidence of gestational diabetes mellitus, Directorate-General of Health - International Association of Diabetes and Pregnancy Study Groups criteria may be associated with greater healthcare-related costs due to more frequent consultations, with no apparent obstetrical/neonatal benefit.

Conclusion: The Directorate-General of Health - International Association of Diabetes and Pregnancy Study Groups criteria were associated with a decrease in macrosomia, not accompanied by an improvement of obstetrical/perinatal outcomes. The benefit of using these criteria is open to debate.

Keywords: Diabetes, Gestational/diagnosis; Diabetes, Gestational/epidemiology; Portugal

\footnotetext{
* Os autores contribuíram de igual forma para a elaboração do presente trabalho.

1. Serviço de Obstetrícia A. Maternidade Daniel de Matos. Centro Hospitalar e Universitário de Coimbra. Coimbra. Portugal.

2. Faculdade de Medicina da Universidade de Coimbra. Coimbra. Portugal.

$\triangle$ Autor correspondente: Ana Filipa Ferreira. filipaferreira44@gmail.com

Recebido: 22 de Dezembro de 2017 - Aceite: 11 de Junho de 2018 | Copyright @ Ordem dos Médicos 2018
} 


\section{INTRODUÇÃO}

A diabetes gestacional (DG) é definida por uma anomalia da tolerância aos hidratos de carbono diagnosticada ou detetada pela primeira vez durante a gravidez, resultando em graus variáveis de hiperglicémia materna. Esta entidade foi associada a várias complicações obstétricas e perinatais, particularmente a macrossomia fetal, parto traumático e complicações hipertensivas. ${ }^{1}$ Estas complicações podem ser prevenidas através de estratégias de intervenção, que incluem as não-farmacológicas (alterações do estilo de vida, incluindo dieta e exercício físico) e as farmacológicas (insulina e/ou antidiabéticos orais). ${ }^{2}$

O principal desafio é identificar corretamente as grávidas que irão beneficiar da instituição destas estratégias. Desde o seu reconhecimento, têm sido aplicados diferentes critérios de rastreio e diagnóstico da DG. Os primeiros critérios foram os de O'Sullivan e Mahan (1964), ${ }^{3}$ com base no risco para o desenvolvimento de diabetes após a gravidez, e incluíam uma prova de tolerância à glicose oral (PTGO). Em 1982, Carpenter e Coustan 4 modificaram estes critérios, com valores de cut-off intermédios entre os de O'Sullivan e Mahan e os da National Diabetes Data Group (NDDG). Estes critérios foram amplamente utilizados nos Estados Unidos da América (EUA) e em alguns países europeus, incluindo Portugal, até ao início de $2011 .{ }^{5}$ Consistiam numa abordagem a dois passos, com uma prova de rastreio com $50 \mathrm{~g}$ de glicose oral seguida de uma prova diagnóstica com $100 \mathrm{~g}$ de glicose oral na presença de um teste de rastreio positivo. A avaliação da glicémia era realizada às $0 \mathrm{~h}, 1 \mathrm{~h}, 2 \mathrm{~h}$ e $3 \mathrm{~h}$. Paralelamente a estes critérios, a Organização Mundial de Saúde (OMS) (1999)6 recomendou a abordagem a um passo, com uma prova de $75 \mathrm{~g}$ de glicose e avaliação às 0 h ( $\geq 126$ mg/dL) e 2 h ( $\geq 140$ mg/ $\mathrm{dL})$.

Em 2010, a Associação Internacional do Grupo de Estudos de Diabetes e Gravidez ${ }^{7}$ (International Association of Diabetes and Pregnancy Study Groups - IADPSG) recomendou novos critérios para o diagnóstico e classificação da hiperglicémia na gravidez, tendo por base os resultados de um grande estudo multicêntrico (Hyperglycemia and Adverse Pregnancy Outcome - HAPO) $)^{8}$ que demonstrou a existência de uma relação linear entre a glicémia materna e os resultados maternos e perinatais.

Em Portugal, o Consenso sobre Diabetes e Gravidez ${ }^{9} \mathrm{e}$ suas posteriores atualizações, ${ }^{10,11}$ recomenda a utilização dos critérios de diagnóstico preconizados pela IADPSG, que serviram igualmente de fundamento à Circular Normativa da Direcção-Geral da Saúde (DGS) publicada em janeiro de 2011. ${ }^{12} \mathrm{O}$ diagnóstico pode ser realizado no primeiro trimestre de gravidez, quando o valor da glicémia em jejum na primeira visita pré-natal for igual ou superior a 92 $\mathrm{mg} / \mathrm{dL}$ [e inferior a $126 \mathrm{mg} / \mathrm{dL}$, caso em que se trata de um diagnóstico de diabetes mellitus (DM) na gravidez]. Caso o valor seja inferior a $92 \mathrm{mg} / \mathrm{dL}$, a grávida deve ser reavaliada entre as 24 - 28 semanas, através de uma PTGO, segundo os critérios apresentados na Tabela 1.

Os critérios da IADPSG foram adotados por outras Sociedades e Organizações internacionais, como a Associação Americana de Diabetes (American Diabetes Association - ADA), ${ }^{13}$ a Federação Internacional de Ginecologia e Obstetrícia (International Federation of Gynecology and Obstetrics - FIGO),${ }^{14}$ a Federação Internacional de Diabetes (International Diabetes Federation - IDF) ${ }^{15}$ e a OMS. ${ }^{1}$ No entanto, com base na relação custo-benefício, várias outras reconhecidas Sociedades adotaram diferentes estratégias para o rastreio e diagnóstico da DG, como o Colégio Americano de Obstetrícia e Ginecologia (American College of Obstetricians and Gynecology $-A C O G)^{16}$ e o Instituto Nacional para Saúde do Reino Unido (National Institute for Health and Care Excellence - NICE). ${ }^{17}$ Com base em estudos realizados na sua população, o Grupo Espanhol de Diabetes e Gravidez (GEDE) ${ }^{18}$ considerou não existir evidência para alterar os seus critérios de diagnóstico, que inclui uma estratégia a dois passos.

Não existem estudos randomizados que comparem os resultados obstétricos e perinatais da DG diagnosticada através dos diferentes critérios na população portuguesa. No estudo retrospetivo de Massa et al, ${ }^{19}$ os autores concluíram que os novos critérios (DGS - IADPSG) estiveram associados a uma redução do número de recém-nascidos grandes para a idade gestacional.

O objetivo deste estudo foi avaliar e comparar a incidência de DG, o movimento assistencial da consulta e os resultados obstétricos e perinatais das grávidas diagnosticadas com DG segundo os critérios de Carpenter e Coustan e os critérios da DGS - IADPSG.

\section{MATERIAL E MÉTODOS \\ Desenho do estudo}

Foi realizada a análise retrospetiva da informação clínica de todas as gestações únicas complicadas com DG,

Tabela 1 - Critérios de diagnóstico de DG segundo Carpenter e Coustan e a DGS - IADPSG

\begin{tabular}{ccc}
\hline & Carpenter e Coustan ${ }^{*}$ & DGS - IADPSG \\
Hora após ingestão* & Glicémia plasmática mg/dL (mmol/L) \\
\hline 0 & $95(5,3)$ & $92(5,1)$ \\
1 & $180(10,0)$ & $180(10,0)$ \\
2 & $155(8,6)$ & $153(8,5)$ \\
3 & $140(7,8)$ & \\
\hline
\end{tabular}

* Ingestão de solução com $100 \mathrm{~g}$ de glicose em $400 \mathrm{cc}$ de água e avaliação segundo os critérios de Carpenter e Coustan ou ingestão de solução com $75 \mathrm{~g}$ de glicose em 250 - 300 cc de água e avaliação segundo os critérios DGS-IADPSG. It A prova é considerada positiva quando $\geq 2$ valores são atingidos ou ultrapassados. ${ }^{\Uparrow} \mathrm{A}$ prova é considerada positiva quando $\geq 1$ valor é atingido ou ultrapassado 
vigiadas num hospital terciário, cujo parto ocorreu na mesma instituição entre os anos de 2008 e 2015. Para evitar um viés de informação, dado existir uma quantidade considerável de registos incompletos relativos ao ano de 2013, foram excluídos todos os dados relativos a este ano. Assim, a amostra final diz respeito a sete anos.

\section{Grupos de estudo}

Entre 2008 e 2011, o diagnóstico de DG foi estabelecido através de uma estratégia a dois passos: realização de uma prova de rastreio, com a ingestão de $50 \mathrm{~g}$ de glicose entre as 24 e 28 semanas (ou no primeiro trimestre em muIheres com fatores de risco, assim como às 32 semanas em grávidas com rastreio negativo até ao momento); as mulheres com glicémia igual ou superior a $140 \mathrm{mg} / \mathrm{dL}$ após uma hora, realizaram posteriormente uma PTGO com 100 $\mathrm{g}$ de glicose. O diagnóstico de DG foi realizado com base nos critérios de Carpenter e Coustan, conforme descrito na Tabela 1.

A partir de 2011, com a atualização dos critérios da DGS - IADPSG para o diagnóstico de DG, esta passou a ser diagnosticada através da ocorrência de um valor de glicémia em jejum igual ou superior a $92 \mathrm{mg} / \mathrm{dL}$ em qualquer momento da gestação, ou de alteração da PTGO com $75 \mathrm{~g}$ de glicose, realizada entre as 24 e 28 semanas, conforme descrito na Tabela 1.

Os dados foram analisados considerando dois grupos:

- Grupo 1, constituído por grávidas diagnosticadas pelos critérios mais recentes (DGS - IADPSG);

- Grupo 2, constituído por grávidas diagnosticadas através dos critérios de Carpenter e Coustan (CC).

\section{Vigilância e terapêutica da DG}

A vigilância das gestações complicadas por DG foi realizada numa consulta especializada, por uma equipa multidisciplinar incluindo Obstetras, Endocrinologistas, Nutricionistas e Enfermeiros. Para esta consulta são referenciadas, na grande maioria, grávidas com DG, embora também sejam referenciadas grávidas com DM tipo 1 e 2 e outras patologias endocrinológicas, sobretudo patologia tiroideia.

O controlo metabólico durante a gravidez foi considerado adequado quando se verificaram valores de glicémia em jejum entre 60 e 90 mg/dL e de glicémia pós-prandial (após uma hora) entre 100 e $120 \mathrm{mg} / \mathrm{dL}$. Nos casos em que estes objetivos não foram atingidos em uma a duas semanas através de medidas dietéticas e exercício físico, foi instituída terapêutica com insulina. A abordagem terapêutica manteve-se a mesma durante todo o período em estudo, mesmo após a alteração dos critérios de diagnóstico.

\section{Outcomes metabólicos, obstétricos e perinatais}

Num primeiro momento, foi estudada a incidência de DG para cada grupo [(número de novos casos de DG por ano) / (número total de partos por ano)] e o número de primeiras consultas e consultas subsequentes de Endocrinologia/Obstetrícia realizadas na instituição. Assumindo que a referenciação por outras patologias se manteve estável ao longo dos anos, os autores entenderam poder inferir, com alguma margem de erro, o impacto dos mais recentes critérios de DG no número de consultas.

Seguidamente, a amostra foi caracterizada relativamente à idade, paridade, índice de massa corporal (IMC) prévio à gravidez, hemoglobina glicada $(\mathrm{HbA} 1 \mathrm{c})$ no primeiro trimestre, antecedentes patológicos, obstétricos e familiares relevantes e momento do diagnóstico de DG (primeiro ou segundo/terceiro trimestre).

A avaliação de outcomes metabólicos foi realizada através da avaliação dos seguintes parâmetros: necessidade de instituição de terapêutica com insulina, assim como a idade gestacional em que foi instituída; ganho ponderal ao longo da gestação; HbA1c no terceiro trimestre. O aumento de peso ao longo da gravidez foi classificado em adequado, insuficiente ou excessivo de acordo com as recomendações do Consenso de Diabetes e Gravidez. ${ }^{9}$

A avaliação de outcomes obstétricos e perinatais foi realizada através da avaliação dos seguintes parâmetros: taxa de cesariana (todas as causas), parto pré-termo, parto instrumentado, complicações hipertensivas, peso e classificação do peso do recém-nascido (RN), APGAR ao $1^{\circ}$ e $5^{\circ}$ minuto, complicações do parto (distócia de ombros, fratura da clavícula, lesão do plexo braquial), necessidade de internamento em Unidade de Cuidados Intensivos do RN (UCIRN), morbilidade neonatal (síndrome de dificuldade respiratória, hipoglicémia e hiperbilirrubinémia com necessidade de fototerapia), malformações major do RN (definidas como as que condicionam defeito funcional ou cosmético significativo, as que requerem cirurgia ou as que colocam a vida em risco) e resultados obtidos na prova de reclassificação pós-parto (PTGO com sobrecarga de $75 \mathrm{~g}$ de glicose com determinação de glicémia às $0 \mathrm{~h}$ e $2 \mathrm{~h}$ ). Os $\mathrm{RN}$ com peso inferior ao percentil 10 para a idade gestacional foram considerados leves para a idade gestacional (LIG) e os que apresentavam peso superior ao percentil 90 foram considerados grandes para a idade gestacional (GIG). Um peso igual ou superior a $4000 \mathrm{~g}$ definiu os $\mathrm{RN}$ como macrossómicos.

\section{Análise estatística}

Os grupos 1 e 2 foram comparados através do teste $t$ de Student e qui-quadrado/ teste exato de Fisher para as variáveis continuas e categóricas, respetivamente. A associação entre variáveis categóricas foi avaliada através do cálculo do odds ratio (OR) e risco relativo ( $\mathrm{RR})$.

Os resultados relativos à classificação do peso do RN conduziram a uma análise posterior, com o objetivo de estudar os preditores de RN LIG e GIG. Com base na análise univariada, foi realizada uma análise multivariada para identificar os parâmetros independentes relacionados com a ocorrência de RN LIG e GIG. Foram calculados os OR ajustados através de modelos de regressão logística.

A análise estatística foi efetuada com recurso ao programa IBM SPSS Statistics ${ }^{\circledR}$ 20.0. Considerou-se como estatisticamente significativo um valor de $p<0,05$. 


\section{RESULTADOS}

A incidência de DG aumentou ligeiramente entre 2008 e 2010, e aumentou consideravelmente nos anos subsequentes, após a aplicação dos critérios da DGS - IADPSG (Fig. 1). A incidência em 2015 (9,4\%) foi aproximadamente o dobro da verificada em 2008 (4,6\%). De forma semelhante, o número total consultas de Endocrinologia/Obstetrícia aumentou ligeiramente entre 2008 e 2010 e consideravelmente a partir de 2011, após a instituição dos critérios atuais de diagnóstico de DG. O número de primeiras consultas de Endocrinologia/Obstetrícia manteve-se igual nos dois anos após a aplicação dos critérios atuais e aumentou ligeiramente nos anos 2014-2015 (Fig. 1).

De um total de 1218 mulheres diagnosticadas com DG, em 660 casos $(54.2 \%)$ o diagnóstico foi feito segundo os critérios da DGS - IADPSG (grupo 1) e em 558 casos $(45,8 \%)$ segundo os critérios de Carpenter e Coustan (grupo 2). O diagnóstico foi realizado no primeiro trimestre em $45,2 \%$ dos casos no grupo 1 e em $3,6 \%$ dos casos no grupo $2(p<0,0001)$ (Tabela 2).

Os grupos eram semelhantes, com exceção dos antecedentes pessoais de DG, mais prevalentes no grupo 2, e um maior número de mulheres com baixo peso no grupo 1 .

Os resultados da comparação de outcomes metabólicos encontram-se na Tabela 3. Em média, as grávidas do grupo 1 tiveram um ganho ponderal de menos $1,4 \mathrm{~kg}$ que as do grupo $2(p<0,0001)$. A classificação do ganho ponderal em relação ao IMC pré-concecional foi diferente entre grupos $(p<0,0001)$. A percentagem de mulheres com um ganho ponderal adequado para o IMC prévio foi ligeiramente superior no grupo $2(37,8 \%$ vs $30,1 \%)$. No grupo 1, cerca de metade das grávidas $(50,5 \%)$ teve um ganho ponderal insuficiente. No grupo 2, em $27,8 \%$ das mulheres houve um ganho ponderal excessivo. $\mathrm{O}$ valor médio de $\mathrm{HbA} 1 \mathrm{c}$ no terceiro trimestre foi $0,3 \%$ inferior no grupo $1(p<0,0001)$. A necessidade de terapêutica com insulina foi significativamente superior no grupo 1 , e a sua instituição mais precoce.

$\mathrm{Na}$ Tabela 4 estão apresentados os dados relativos à comparação de outcomes obstétricos e perinatais. A frequência de RN macrossómicos $(3,0 \%$ vs $6,8 \%, p=0,002)$ e GIG $(10,0 \%$ vs $18,5 \% p<0,0001)$ foi inferior no grupo 1 . O risco de uma grávida do grupo 1 ter um filho macrossómico foi cerca de metade [RR 0,44 (IC 95\%: 0,26 - 0,76)] em relação a uma grávida do grupo 2. Contrariamente, o risco de ter um filho LIG foi duas vezes superior no grupo 1 [RR 1,99 (IC 95\%: 1,19 - 3,31)]. No grupo 1 houve uma maior frequência de complicações neonatais, com um risco seis vezes superior de hipoglicémia neonatal [RR 6,30 (IC 95\%: 3,39 - 11,71)] e quatro vezes superior de hiperbilirrubinémia [RR 6,30 (IC 95\%: 3,39b - 11,70)]. A taxa de cesariana foi inferior no grupo $1(31,1 \%$ vs $37,5 \%, p=0,019)$. Esta diferença decorre da indicação "Indução Falhada", não existindo diferenças entre grupos em relação à taxa de cesariana por suspeita de macrossomia e incompatibilidade feto-péIvica. Não houve diferenças significativas entre os grupos

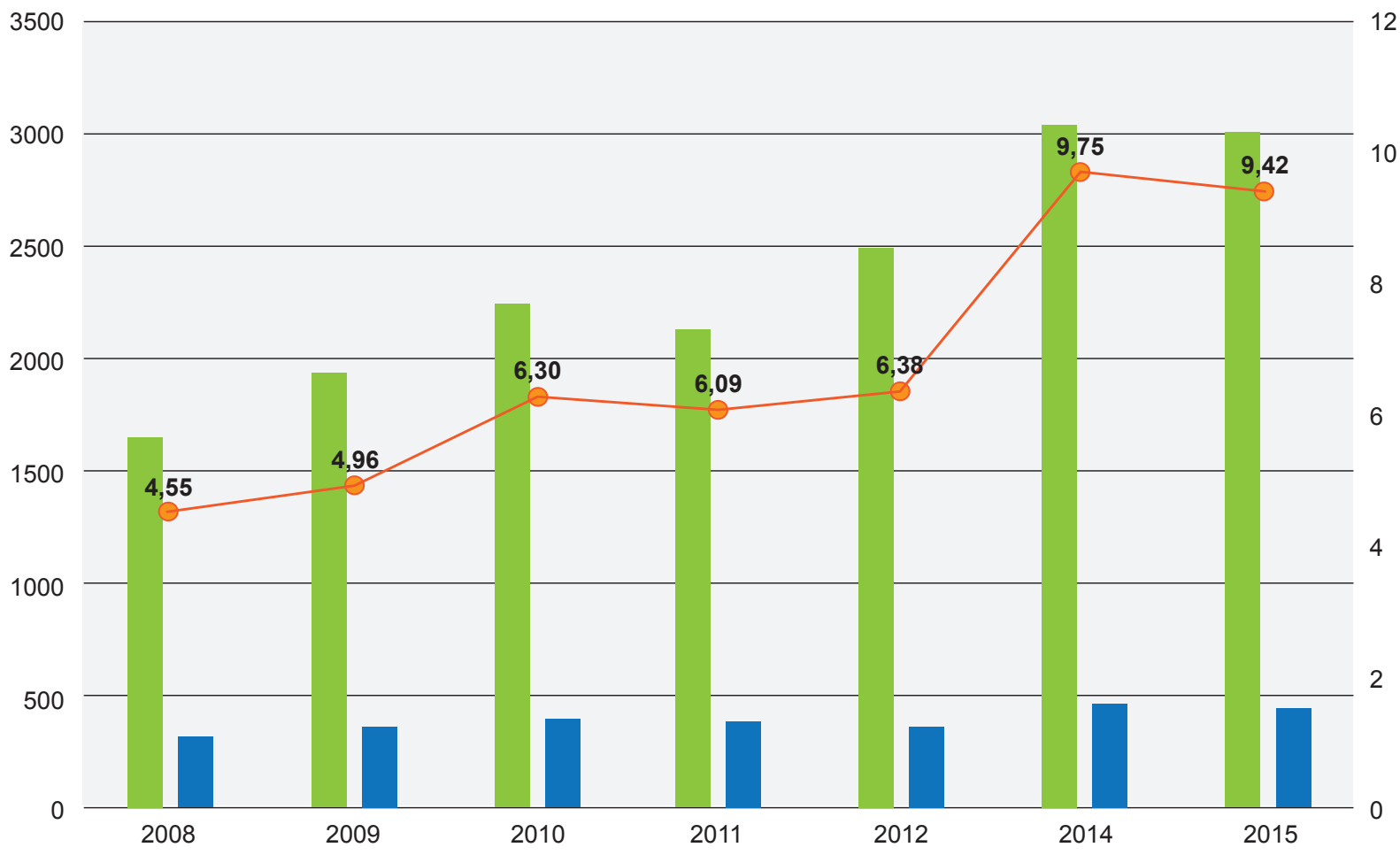

$\mathrm{N}^{\circ}$ de consultas (total)

$\mathrm{N}^{\circ}$ de consultas (primeiras)

Incidência (\%)

Figura 1 - Incidência de DG e Consultas de Endocrinologia/Obstetrícia 
Tabela 2 - Características clínicas das mulheres diagnosticadas com DG por grupo de critérios de diagnóstico

\begin{tabular}{|c|c|c|c|}
\hline & $\begin{array}{c}\text { Grupo } 1 \\
\text { DGS - IADPSG }\end{array}$ & $\begin{array}{c}\text { Grupo } 2 \\
\text { CC }\end{array}$ & \\
\hline & $n=660$ & & \\
\hline & \multicolumn{2}{|c|}{ Média \pm DP ou frequência (\%) } & $p$ \\
\hline Idade & $33,0 \pm 4,9$ & $33,0 \pm 4,7$ & 0,877 \\
\hline IMC pré-concecional & $26,1 \pm 5,3$ & $26,5 \pm 5,3$ & 0,149 \\
\hline \multicolumn{4}{|c|}{ Classificação segundo IMC pré-concecional " } \\
\hline Baixo peso & $2,0^{*}$ & $0,6^{*}$ & \multirow{4}{*}{0,147} \\
\hline Normal & 47,8 & 45,5 & \\
\hline Excesso de peso & 29,4 & 31,4 & \\
\hline Obesidade & 20,8 & 22,5 & \\
\hline Primigesta & 38,0 & 38,4 & 0,909 \\
\hline HTA crónica & 4,2 & 4,1 & 0,917 \\
\hline Antecedentes pessoais de DG & 17,0 & 25,4 & $0,010^{*}$ \\
\hline Antecedentes familiares de DM & 45,1 & 50,2 & 0,079 \\
\hline Diagnóstico no $1^{\circ}$ trimestre & 45,2 & 3,6 & $<0,0001^{*}$ \\
\hline $\mathrm{HbA} 1 \mathrm{c}$ no $1^{\circ}$ trimestre & $5,2 \pm 0,3$ & $5,4 \pm 0,5$ & 0,252 \\
\hline
\end{tabular}

* Diferenças significativas para o nível de significância de 0,05. "Baixo peso (IMC $\left.<18,5 \mathrm{~kg} / \mathrm{m}^{2}\right)$, Normal $\left(18,5 \mathrm{~kg} / \mathrm{m}^{2} \leq \mathrm{IMC}<25,0 \mathrm{~kg} / \mathrm{m}^{2}\right)$, Excesso de peso $\left(25,0 \mathrm{~kg} / \mathrm{m}^{2} \leq \mathrm{IMC}<30,0\right.$ $\left.\mathrm{kg} / \mathrm{m}^{2}\right)$, Obesidade $\left(\mathrm{IMC} \geq 30,0 \mathrm{~kg} / \mathrm{m}^{2}\right)$.

DP: desvio padrão, IMC: índice de massa corporal, HTA: hipertensão arterial, DG: diabetes gestacional, DM: diabetes mellitus, HbA1c: hemoglobina glicada

em relação a complicações hipertensivas, parto pré-termo e instrumentado, complicações do parto, malformações do RN, número de internamentos na UCIRN e APGAR ao primeiro e quinto minutos.

A prova de reclassificação pós-parto diagnosticou DM $(0,4 \%$ vs $0,6 \%)$, anomalia da glicémia em jejum $(0,9 \%$ vs $0,6 \%)$ e tolerância diminuída à glicose $(3,9 \%$ vs $3,4 \%)$ em percentagens muito semelhantes. Em $94,8 \%$ e $95,3 \%$ dos casos verificou-se um diagnóstico final de DG no grupo $1 \mathrm{e}$ 2, respetivamente.

Os resultados relativos ao peso dos RN conduziram ao estudo dos parâmetros preditivos de RN LIG e GIG. Inicialmente, foi realizada uma análise univariada, comparando as médias e estudando associações entre a ocorrência de RN LIG e GIG e as seguintes variáveis: idade materna, IMC pré-concecional, obesidade, ganho ponderal na gravidez, hipertensão arterial crónica, $\mathrm{HbA} 1 \mathrm{c}$ no primeiro e terceiro trimestres, PTGO às $0 \mathrm{~h}, 1 \mathrm{~h}$ e $2 \mathrm{~h}$ e necessidade de terapêutica com insulina (dados não mostrados). Posteriormente, foram identificados os preditores para RN GIG e LIG e calculados os OR ajustados.

Como exposto na Tabela 5, a obesidade, o ganho ponderal na gravidez e a HbA1c no terceiro trimestre foram fatores preditores independentes para RN GIG. A obesidade esteve associada a uma ocorrência 3,2 vezes superior de RN GIG. Mulheres mais velhas e com um menor ganho ponderal durante a gravidez apresentaram uma maior ocorrência de RN LIG. Este risco foi 3,8 vezes superior por cada unidade a menos de $\mathrm{HbA} 1 \mathrm{c}$ no terceiro trimestre.

\section{DISCUSSÃO}

Na nossa instituição, a aplicação dos critérios de diagnósticos da DGS - IADPSG resultou numa duplicação da incidência de DG (4,6\% vs 9,4\%), como previsto por vários

Tabela 3 - Comparação de outcomes metabólicos por grupo de critérios de diagnóstico

\begin{tabular}{|c|c|c|c|}
\hline & $\begin{array}{c}\text { Grupo } 1 \\
\text { DGS - IADPSG } \\
n=660\end{array}$ & $\begin{array}{c}\text { Grupo } 2 \\
\text { CC } \\
n=558\end{array}$ & \\
\hline & \multicolumn{2}{|c|}{ Média \pm DP ou Frequência (\%) } & $p$ \\
\hline Ganho ponderal na gravidez (kg) & $9,0 \pm 6,2$ & $10,4 \pm 5,2$ & $<0,0001^{*}$ \\
\hline \multicolumn{4}{|l|}{ Classificação do ganho ponderal } \\
\hline Adequado & 30,1 & 37,8 & \\
\hline Insuficiente & 50,5 & 34,4 & $<0,0001^{*}$ \\
\hline Excessivo & 18,4 & 27,8 & \\
\hline $\mathrm{HbA} 1 \mathrm{c}$ no $3^{\circ}$ trimestre $(\%)$ & $5,3 \pm 0,5$ & $5,6 \pm 0,7$ & $<0,0001^{*}$ \\
\hline IG de início de terapêutica com insulina & $25,7 \pm 8,2$ & $31,8 \pm 5,4$ & $<0,0001^{*}$ \\
\hline Necessidade de terapêutica com insulina & 54,9 & 35,9 & $<0,0001^{*}$ \\
\hline
\end{tabular}

* Diferenças significativas para o nível de significância de 0,05

DP: desvio padrão; IG: idade gestacional; HbA1c: hemoglobina glicada 
Tabela 4 - Comparação de outcomes obstétricos e perinatais por grupo de critérios de diagnóstico

\begin{tabular}{|c|c|c|c|c|}
\hline & $\begin{array}{c}\text { Grupo } 1 \\
\text { DGS - IADPSG } \\
n=660\end{array}$ & $\begin{array}{c}\text { Grupo } 2 \\
\text { CC } \\
n=558\end{array}$ & & \\
\hline & \multicolumn{2}{|c|}{ Frequência (\%) } & $p$ & $\operatorname{RR}(95 \%$ IC) \\
\hline RN macrossómicos & $20(3,0)$ & $38(6,8)$ & $0,002^{*}$ & $0,44(0,26-0,76)$ \\
\hline RN GIG & $66(10,0)$ & $103(18,5)$ & $<0,0001^{*}$ & $0,54(0,41-0,72)$ \\
\hline RN LIG & $47(7,1)$ & $20(3,6)$ & $0,007^{*}$ & $1,99(1,19-3,31)$ \\
\hline Complicações do parto" & $8(1,2)$ & $12(2,2)$ & 0,199 & $0,56(0,23-1,37)$ \\
\hline Parto instrumentado & $149(22,6)$ & $130(23,3)$ & 0,763 & $0,97(0,79-1,19)$ \\
\hline Cesariana & $205(31,1)$ & $209(37,5)$ & $0,019^{*}$ & $0,83(0,71-0,97)$ \\
\hline Suspeita de macrossomia & $20(9,8)$ & $25(12,0)$ & & \\
\hline EFNT & $48(23,4)$ & $62(29,7)$ & & \\
\hline NPD/IFP & $46(22,4)$ & $42(20,1)$ & & \\
\hline Apresentação pélvica & $31(15,1)$ & $38(18,2)$ & 0,128 & \\
\hline Indicação materna & $17(8,3)$ & $11(5,3)$ & & \\
\hline Indução falhada & $20(9,8)^{*}$ & $8(3,8)^{*}$ & & \\
\hline Outro motivo & $19(9,3)$ & $16(7,7)$ & & \\
\hline Hipoglicémia neonatal & $82(12,4)$ & $11(2,0)$ & $<0,0001^{*}$ & $6,30(3,39-11,71)$ \\
\hline Complicações hipertensivas & $15(2,3)$ & $8(1,4)$ & 0,284 & $1,58(0,68-3,71)$ \\
\hline Parto pré-termo & $47(7,1)$ & $44(7,9)$ & 0,613 & $0,90(0,61-1,34)$ \\
\hline SDR neonatal & $18(2,7)$ & $10(1,8)$ & 0,278 & $1,52(0,71-3,27)$ \\
\hline Hiperbilirrubinémia neonatal & $69(10,5)$ & $15(2,7)$ & $<0,0001^{*}$ & $3,89(2,25-6,72)$ \\
\hline Internamentos na UCIRN & $35(5,4)$ & $19(3,4)$ & 0,101 & $1,57(0,91-2,72)$ \\
\hline Apgar ao $1^{\prime}<7$ & $40(6,1)$ & $37(6,7)$ & 0,687 & $0,92(0,59-1,41)$ \\
\hline Apgar ao $5^{\prime}<7$ & $1(0,2)$ & $4(0,7)$ & 0,124 & $0,21(0,02-1,89)$ \\
\hline Malformação major do RN \# & $27(5,1)$ & $18(3,7)$ & 0,284 & $1,37(0,77-2,46)$ \\
\hline
\end{tabular}

* Diferenças significativas para o nível de significância de 0,05. ๆ Distócia de ombros, fratura da clavícula, lesão do plexo braquial. t Condiciona defeito funcional ou cosmético significativo.

DP: desvio padrão; RN: recém-nascido; GIG: grande para a idade gestacional; LIG: leve para a idade gestacional; EFNT: estado fetal não tranquilizador; NPD/IFP: não progressão da dilatação/incompatibilidade feto-pélvica; SDR: síndrome de dificuldade respiratória; UCIRN: Unidade de cuidados intensivos do recém-nascido RR: risco relativo

autores $\mathrm{s}^{7,20}$ e verificado em estudos experimentais ${ }^{21,22}$ e observacionais. ${ }^{19,23} \mathrm{~A}$ incidência mundial de DG situa-se entre $1 \%$ e $14 \%,{ }^{22}$ sendo muito variável devido à falta de consenso internacional nos critérios de diagnóstico. $\mathrm{O}$ aumento da incidência no nosso estudo deveu-se sobretudo à realização de diagnósticos no primeiro trimestre, pouco frequentes com os critérios anteriores $(45,2 \%$ vs $3,6 \%)$, à semeIhança do observado no estudo de Massa et al (30,8\% vs $1,8 \%)$, também realizado numa maternidade portuguesa.

Dado o aumento global da prevalência de obesidade e DM na população mais jovem, a pesquisa da glicémia em jejum no primeiro trimestre tem como objetivo identificar mulheres com DM previamente não diagnosticadas. Neste sentido, a pesquisa universal ou apenas em mulheres com fatores de risco foi debatida pelo painel de peritos da IADPSG, ${ }^{7}$ que reconheceu a importância de conhecer a prevalência específica de DM na população jovem em causa. Não obstante, o painel concluiu não existir evidência suficiente para recomendar o diagnóstico e tratamento da DG antes das 24 - 28 semanas. A pesquisa da glicémia em jejum na primeira consulta pré-natal com o objetivo de identificar e tratar DG não é, assim, a conduta

Tabela 5 - Preditores independentes de recém-nascidos grandes para a idade gestacional (GIG) e leves para a idade gestacional (LIG)

\begin{tabular}{|c|c|c|c|c|}
\hline & \multicolumn{4}{|c|}{ aOR (95\% do IC) } \\
\hline & $\mathrm{GIG}^{*}$ & $p$ & $\mathrm{LIG}^{\star *}$ & $p$ \\
\hline Obesidade & $3,209(1,782-5,780)$ & $<0,0001$ & - & \\
\hline Ganho ponderal na gravidez $(\mathrm{kg})$ & $1,061(1,011-1,113)$ & 0,016 & $0,908(0,826-0,998)$ & 0,046 \\
\hline $\mathrm{HbA} 1 \mathrm{c}$ no $3^{\circ}$ trimestre $(\%)$ & $2,525(1,322-4,823)$ & 0,005 & $0,261(0,083-0,823)$ & 0,022 \\
\hline Idade (anos) & - & & $1,146(1,033-1,270)$ & 0,010 \\
\hline
\end{tabular}

* OR foram ajustados para as seguintes covariáveis: PTGO às $0 \mathrm{~h}, 1 \mathrm{~h}$ e $2 \mathrm{~h}$, obesidade, ganho ponderal na gravidez, HbA1c no $3^{\circ}$ trimestre e terapêutica com insulina. ** OR foram ajustados para as seguintes covariáveis: idade materna, classe de IMC pré-concecional, ganho ponderal na gravidez e HbA1c no $3^{\circ}$ trimestre.

aOR: odds ratio ajustado; IC: intervalo de confiança 
recomendada por várias reconhecidas Sociedades, ${ }^{13,16,17}$ sendo até contraditória com a declaração da $A D A,{ }^{13}$ que define DG como a diabetes que é diagnosticada no segundo e terceiro trimestres da gravidez. De acrescentar que o mecanismo fisiopatológico responsável pela DG parece resultar de um aumento não fisiológico da insulinorresistência, que se inicia habitualmente às 20 - 24 semanas. A causa permanece por esclarecer, podendo a insulinorresistência ser explicada pela unidade feto-placentária, nomeadamente o aumento na segunda metade da gravidez do lactogénio placentário humano, ou pelo tecido adiposo materno, sabendo-se que certas adiponectinas alteram a sensibilidade à insulina. ${ }^{24}$

No nosso estudo, como também verificado por Massa et $a l,{ }^{19}$ houve um número reduzido de mulheres com diagnóstico de DM na prova de reclassificação pós-parto, sem diferenças entre critérios $(0,4 \%$ vs $0,6 \%)$. Em Portugal, a prevalência de DM em mulheres jovens (20 - 39 anos) no ano de 2015 foi de $1,5 \% .{ }^{25}$ Assim, com o intuito de identificar a Diabetes prévia, dada a reduzida prevalência da doença, seria de considerar a pesquisa seletiva da glicémia em jejum na primeira consulta pré-natal, apenas em muIheres com fatores de risco. Esta estratégia de rastreio seletivo, com base em fatores de risco, foi também estudada para o diagnóstico entre as 24 - 28 semanas por Miailhe, et al. Estes autores concluíram que, apesar de um sexto dos casos de DG escaparem ao diagnóstico, estes seriam de mulheres com valores glicémicos ligeiramente elevados e não associados a complicações.

Os gastos em saúde com a implementação dos critérios IADPSG têm sido estudados por alguns autores, que concluíram existir custo-efetividade. ${ }^{23,26}$ No entanto, numa revisão da NICE, ${ }^{17}$ que inclui cinco estudos, os autores concluíram que os potenciais benefícios da introdução dos critérios IADPSG só seriam atingidos com um incremento de custo não aceitável. Além disso, segundo uma revisão recente da Cochrane Collaboration, ${ }^{21}$ não existem estudos abrangentes que comparem os gastos em saúde com a aplicação de diferentes estratégias de diagnóstico de DG. Não foi objetivo deste trabalho estudar o custo-efetividade da aplicação dos critérios DGS - IADPSG em relação aos anteriores. No entanto, verificámos um aumento considerável do número de consultas de acompanhamento destas grávidas ( 3000 vs 2000) assim como um aumento da terapêutica com insulina (54,9 \% vs 35,9\%), não acompanhados de uma melhoria dos outcomes neonatais (número de internamentos na UCIRN, malformações do RN) e mesmo com aumento de algumas complicações (hipoglicémia e hiperbilirrubinémia). Verificou-se também uma diminuição da taxa global de cesariana, não sendo, contudo, possível atribuir este resultado à utilização de diferentes critérios, dado que não ocorreram diferenças na realização de cesarianas por suspeita de macrossomia ou incompatibilidade feto-pélvica.

No nosso estudo, a utilização dos critérios de diagnóstico da DGS - IADPSG permitiu atingir um dos grandes objetivos do diagnóstico e tratamento da DG, observando-se uma redução de cerca de $50 \%$ dos RN macrossómicos e GIG. Em paralelo, neste grupo verificou-se um maior controlo metabólico, com valores inferiores de $\mathrm{HbA} 1 \mathrm{c}$ no $3^{\circ}$ trimestre, menor ganho ponderal e utilização mais frequente e mais precoce de insulinoterapia, que poderá explicar os resultados referidos. No entanto, verificou-se simultaneamente uma duplicação dos RN LIG em relação aos anteriores critérios de diagnóstico. Para melhor esclarecer estes resultados, foram estudados os fatores preditivos independentes de RN GIG e LIG, ajustados para fatores de confundimento.

Independentemente do valor da PTGO às 0 horas, 1 hora e 2 horas, a obesidade foi um fator preditor de RN GIG, resultado suportado em vários trabalhos. ${ }^{24,27,28}$ As mulheres obesas tiveram uma incidência 3,2 vezes superior de RN GIG. A HbA1c no terceiro trimestre e o ganho ponderal durante a gravidez também se associaram a RN GIG, com OR inferiores. Segundo o modelo de fisiopatologia baseado na hipótese de Pedersen modificada, o maior crescimento fetal é consequência da hiperglicémia materna, por intermédio da hiperinsulinémia fetal. ${ }^{24}$ No entanto, a macrossomia fetal ocorre mesmo na presença de um excelente controlo metabólico ${ }^{29}$ e, no estudo HAPO, a maioria dos casos de GIG ocorreu em categorias de glicémia normais. ${ }^{30}$ Uma explicação poderá ser a presença de valores elevados de triglicerídeos, segundo os resultados de Son et $a l,{ }^{31}$ que concluiu que a hipertrigliceridémia é um fator preditivo independente de RN GIG.

Apesar destas considerações, a redução dos RN GIG e macrossómicos no nosso estudo parece ter sido consequência do controlo dos níveis de glicémia, tendo em conta que a percentagem de mulheres obesas e com excesso de peso foi semelhante entre grupos. De igual forma, o aumento de RN LIG poderá ter sido consequência da diminuição dos níveis de glicémia, com uma incidência 3,8 vezes superior por cada unidade a menos de HbA1C no terceiro trimestre. A idade materna e o ganho ponderal também foram preditores de RN LIG, o que está de acordo com o descrito noutros estudos. ${ }^{32,33}$ De salientar que no grupo 1 (DGS - IADPSG), metade das mulheres teve um ganho ponderal inferior ao recomendado. Na análise multivariada realizada, as variáveis não foram ajustadas para o tabagismo, o que constitui uma limitação, uma vez que este é um importante fator de risco para RN LIG. ${ }^{33}$

Associada à redução dos RN macrossómicos e GIG no grupo diagnosticado com os critérios da DGS - IADPSG, seria expectável a melhoria de outcomes obstétricos e perinatais relacionados, particularmente a redução da taxa de cesariana e parto instrumentado, diminuição das complicações do parto (distócia de ombros, fratura da clavícula, lesão do plexo braquial) e diminuição da hipoglicémia e hiperbilirrubinémia neonatais, assim como o número de internamentos na UCIRN.

A diminuição da taxa de cesariana global (por todas as causas) verificada não se pode atribuir diretamente à redução dos RN macrossómicos tendo em conta a análise das indicações de cesariana (ausência de diferenças na taxa 
de cesariana por suspeita de macrossomia e incompatibilidade feto-pélvica). Assim, contrariamente ao que seria de prever, não houve melhoria nos outcomes e houve até aumento das complicações neonatais, observando-se um risco seis vezes superior de hipoglicémia e quatro vezes superior de hiperbilirrubinémia. Estes resultados são também contraditórios ao que seria expectável. A hipoglicémia poderá ser explicada pelo hiperinsulinismo adquirido associado a RN LIG ${ }^{34}$. A hiperbilirrubinémia poderá relacionar-se com a policitémia secundária ao aumento da eritropoiese, também associada a RN LIG. ${ }^{35}$

Além dos outcomes mencionados, os critérios DGS IADPSG não estiveram associados a redução dos partos pré-termo, partos instrumentados, complicações hipertensivas e malformações do RN.

Apesar das recomendações do grupo de trabalho da IADPSG, suportadas pelos resultados do estudo HAPO, continua a não existir consenso internacional sobre o rastreio e diagnóstico da DG. Reconhecendo tratar-se de um grande ensaio prospetivo multicêntrico, foram apontadas algumas questões às orientações clínicas derivadas deste estudo: 1) trata-se de um estudo observacional, não tendo sido investigado o benefício em tratar hiperglicémias moderadas; ${ }^{17}$ 2) a associação entre os resultados adversos e a glicémia materna é linear, pelo que qualquer valor limite utilizado para o diagnóstico de DG é arbitrário, tendo este sido definido por um grupo de peritos como o correspondente a um risco 1,75 vezes superior de resultados perinatais adversos $^{36,37}$; 3) a glicémia e o IMC maternos contribuíram ambos para o risco de RN GIG, mas com um maior impacto do IMC em todas as categorias de glicémia, com exceção da categoria mais alta. ${ }^{30}$

Existem atualmente dois ensaios clínicos randomizados em curso ${ }^{38,39}$ que pretendem comparar os outcomes obstétricos obtidos após o diagnóstico de DG através da abordagem a um passo (conforme proposto pela IADPSG) ou a dois passos. Estes ensaios poderão vir a esclarecer qual a forma de diagnóstico de DG que resulta em melhores resultados materno-fetais.

Assim, as dúvidas de há 15 anos atrás, descritas por Fagulha, ${ }^{40}$ permanecem atuais: qual o melhor método na identificação das grávidas com risco de morbilidade materna e perinatal? Qual o valor glicémico ideal para instituição de dieta e/ou insulina?

O nosso estudo tem as limitações inerentes a um estudo retrospetivo, para além de se tratar de um estudo num único centro. Teria sido interessante realizar uma análise custo-efetividade de forma a estudar o impacto dos mais recentes critérios de diagnóstico de DG nos recursos de saúde. A utilização dos objetivos glicémicos de 60 - 90 mg/ dL em jejum/pré-prandial e 100 - 120 mg/dL uma hora após a refeição poderá ter tido influência nalguns dos resultados obtidos (nomeadamente as diferenças no aumento ponderal gestacional, $\mathrm{HbA1c}$ no terceiro trimestre, necessidade e idade gestacional do início da insulinoterapia, aumento de RN LIG, hipoglicémia e hiperbilirrubinémia neonatal), ao exigir um controlo glicémico restritivo num subgrupo de mulheres com hiperglicémia mais moderada. Atualmente, o Consenso Nacional ${ }^{11}$ recomenda valores glicémicos de $\leq 95 \mathrm{mg} / \mathrm{dL}$ em jejum/pré-prandial e $\leq 140$ mg/dL pós-prandial.

Apesar destas limitações, o nosso estudo baseou-se na comparação de um número significativo de mulheres (cerca de 600 mulheres em cada grupo estudado), que correspondem a todas as mulheres com DG cuja vigilância e parto decorreu na nossa instituição, uma maternidade terciária, orientadas segundo critérios de diagnóstico e abordagem terapêutica uniformes. O facto de os objetivos glicémicos se terem mantido antes e depois da instituição de novos critérios permitiu comparar diretamente os resultados clínicos das diferentes abordagens diagnósticas.

\section{CONCLUSÃO}

O nosso estudo demonstrou que a identificação e tratamento de grávidas com hiperglicémia moderada estiveram associados a uma redução dos RN GIG e macrossómicos. Contudo, esta diminuição não foi acompanhada de uma melhoria dos outcomes obstétricos e perinatais e associou-se mesmo a um aumento dos RN LIG e complicações neonatais. Assim, é discutível o benefício da aplicação dos critérios da DGS-IADPSG em relação aos anteriormente preconizados. Deverão ser realizados estudos prospetivos randomizados neste âmbito, com análise de custo-efetividade, na população portuguesa.

\section{AGRADECIMENTOS}

Os autores agradecem a colaboração da equipa Médica que realiza a Consulta de Obstetrícia/Endocrinologia da Maternidade Daniel de Matos: Elvira Marta, Fernando Jorge Costa, Helena Lopes e Helena Gonçalves (Obstetrícia), Sandra Paiva e Luísa Ruas (Endocrinologia).

\section{PROTECÇÃO DE PESSOAS E ANIMAIS}

Os autores declaram que os procedimentos seguidos estavam de acordo com os regulamentos estabelecidos pelos responsáveis da Comissão de Investigação Clínica e Ética e de acordo com a Declaração de Helsínquia da Associação Médica Mundial.

\section{CONFIDENCIALIDADE DOS DADOS}

Os autores declaram ter seguido os protocolos do seu centro de trabalho acerca da publicação de dados.

\section{CONFLITOS DE INTERESSE}

Os autores declaram não terem qualquer conflito de interesse relativamente ao presente artigo.

\section{FONTES DE FINANCIAMENTO}

Os autores declaram não ter recebido subsídios ou bolsas para a elaboração do artigo. 


\section{REFERÊNCIAS}

1. World Health Organization. Guidelines Approved by the Guidelines Review Committee. Diagnostic Criteria and Classification of Hyperglycaemia First Detected in Pregnancy. Geneva: WHO. 2013.

2. Falavigna M, Schmidt MI, Trujillo J, Alves LF, Wendland ER, Torloni $M R$, et al. Effectiveness of gestational diabetes treatment: a systematic review with quality of evidence assessment. Diabetes Res Clin Pract. 2012;98:396-405.

3. Freinkel N JJ. Summary of Recommendations of the First Workshop Conference on Gestational Diabetes Mellitus. Diabetes Care. 1980;3:499-501.

4. Carpenter MW, Coustan DR. Criteria for screening tests for gestational diabetes. Am J Obstetr Gynecol. 1982;144:768-73.

5. Direção Geral da Saúde. Diabetes e Gravidez. Norma número 08/ DGCG. Lisboa: DGS; 1998.

6. Alberti KG, Zimmet PZ. Definition, diagnosis and classification of diabetes mellitus and its complications. Part 1: diagnosis and classification of diabetes mellitus provisional report of a WHO consultation. Diabet Med. 1998;15:539-53.

7. Metzger BE, Gabbe SG, Persson B, Buchanan TA, Catalano PA Damm $P$, et al. International association of diabetes and pregnancy study groups recommendations on the diagnosis and classification of hyperglycemia in pregnancy. Diabetes Care. 2010;33:676-82.

8. Metzger BE, Lowe LP, Dyer AR, Trimble ER, Chaovarindr U, Coustan $\mathrm{DR}$, et al. Hyperglycemia and adverse pregnancy outcomes. N Engl J Med. 2008;358:1991-2002.

9. Dores J, Almeida MC, Vicente L, Paiva S. Relatório de Consenso sobre a Diabetes e Gravidez. Lisboa: SPEDM, SPD, SPOMMF, SPP: 2011.

10. Almeida MC, Dores J, Vicente L, Paiva S, Ruas L. Consenso "Diabetes Gestacional” - Atualização 2016. Lisboa: Direção Geral da Saúde; 2016.

11. Almeida MC, Dores J, Vicente L, Paiva S, Ruas L. Consenso "Diabetes Gestacional” - Atualização 2017. Lisboa: Direção Geral da Saúde; 2017.

12. Direcção Geral da Saúde. Diagnóstico e Conduta na Diabetes Gestacional. Norma número 007/2011. Lisboa: DGS; 2011.

13. American Diabetes Association. Standards of Medical Care in Diabetes - 2016. Diabetes Care. 2016;39 Suppl 1:S4-5.

14. Hod M, Kapur A, Sacks DA, Hadar E, Agarwal M, Di Renzo GC, et al. The International Federation of Gynecology and Obstetrics (FIGO) Initiative on gestational diabetes mellitus: A pragmatic guide for diagnosis, management, and care. Int J Gynaecol Obstet. 2015;131:S173-211.

15. International Diabetes Federation. GDM Model of care: Implementation protocol, guidelines for healthcare professionals. Brussels: IDF; 2015.

16. Committee on Practice Bulletins-Obstetrics. Practice Bulletin No. 180: Gestational Diabetes Mellitus. Obstet Gynecol. 2017;130:e17-e37.

17. National Collaborating Centre for Ws, Children's H. National Institute for Health and Care Excellence: Clinical Guidelines. Diabetes in Pregnancy: Management of Diabetes and Its Complications from Preconception to the Postnatal Period. London: NICE; 2015.

18. GEDE. Asistencia a la gestante con diabetes. Guía de práctica clínica actualizada en 2014. Grupo Español de Diabetes y Embarazo. Avances Diabetol. 2015;31:45-59.

19. Massa AC, Rangel R, Cardoso M, Campos A. Diabetes gestacional e o impacto do actual rastreio. Acta Med Port. 2015;28:29-34.

20. ADA. Diagnosis and classification of diabetes mellitus. Diabetes Care. 2011;34:S62-9.

21. Farrar D, Duley L, Dowswell T, Lawlor DA. Different strategies for diagnosing gestational diabetes to improve maternal and infant health. Cochrane Database Syst Rev. 2017;8:Cd007122.

22. O'Sullivan EP, Avalos G, O'Reilly M, Dennedy MC, Gaffney G, Dunne F. Atlantic Diabetes in Pregnancy (DIP): the prevalence and outcomes of gestational diabetes mellitus using new diagnostic criteria. Diabetologia. 2011;54:1670-5.

23. Duran A, Saenz S, Torrejon MJ, Bordiu E, Del Valle L, Galindo M, et al. Introduction of IADPSG criteria for the screening and diagnosis of gestational diabetes mellitus results in improved pregnancy outcomes at a lower cost in a large cohort of pregnant women: the St. Carlos Gestational Diabetes Study. Diabetes Care. 2014;37:2442-50.

24. Kc K, Shakya S, Zhang H. Gestational diabetes mellitus and macrosomia: a literature review. Ann Nutr Metab. 2015;66:S14-20.

25. Sociedade Portuguesa de Diabetologia. Factos e Números - O Ano de 2015 - Relatório Anual do Observatório Nacional da Diabetes 2016. Lisboa: SPD; 2016.

26. Werner EF, Pettker CM, Zuckerwise L, Reel M, Funai EF, Henderson J, et al. Screening for gestational diabetes mellitus: are the criteria proposed by the international association of the Diabetes and Pregnancy Study Groups cost-effective? Diabetes Care. 2012;35:529-35.

27. Shin D, Song WO. Prepregnancy body mass index is an independent risk factor for gestational hypertension, gestational diabetes, preterm labor, and small- and large-for-gestational-age infants. J Matern Fetal Neonatal Med. 2015;28:1679-86.

28. Walsh JM, McAuliffe FM. Prediction and prevention of the macrosomic fetus. Eur J Obstet Gynecol Reprod Biol. 2012;162:125-30.

29. Hernandez TL, Friedman JE, Van Pelt RE, Barbour LA. Patterns of glycemia in normal pregnancy: should the current therapeutic targets be challenged? Diabetes Care. 2011;34:1660-8.

30. Ryan EA. Diagnosing gestational diabetes. Diabetologia. 2011;54:4806.

31. Son GH, Kwon JY, Kim YH, Park YW. Maternal serum triglycerides as predictive factors for large-for-gestational age newborns in women with gestational diabetes mellitus. Acta Obstet Gynecol Scand. 2010;89:7004.

32. Campbell MK, Cartier S, Xie B, Kouniakis G, Huang W, Han V. Determinants of small for gestational age birth at term. Paediatr Perinat Epidemiol. 2012;26:525-33.

33. Heaman M, Kingston D, Chalmers B, Sauve R, Lee L, Young D. Risk factors for preterm birth and small-for-gestational-age births among Canadian women. Paediatr Perinat Epidemiol. 2013;27:54-61.

34. Rozance PJ. Update on neonatal hypoglycemia. Curr Opin Endocrinol Diabetes Obes. 2014;21:45-50.

35. Sankar MJ, Agarwal R, Deorari A, Paul VK. Management of polycythemia in neonates. Ind J Pediatr. 2010;77:1117-21.

36. Gouveia C, Granja M, Sá AB, Gomes LF, Simões JA, Gallego R. Novas orientações da Direção-Geral de Saúde para a diabetes gestacional: uma apreciação crítica. Rev Port Med Geral Fam. 2012:304-12.

37. Voormolen DN, Abell SK, James R, Hague WM, Mol BW. Diagnostic Criteria and Treatment for Gestational Diabetes Mellitus. Semin Reprod Med. 2016;34:102-9.

38. Abebe KZ, Scifres C, Simhan HN, Day N, Catalano P, Bodnar LM, Costacou T, Matthew D, Illes A, Orris S, Duell J, Ly K, Davis EM. Comparison of Two Screening Strategies for Gestational Diabetes (GDM2) Trial: Design and rationale. Contemp Clin Trials. 2017;62:43-9.

39. Eunice Kennedy Shriver National Institute of Child Health and Human Development, Kaiser Permanente. Randomizing Two Gestational Diabetes Screening Methods in a Diverse HMO. ClinicalTrials. gov [accessed 2018 May 6]. Available from: https://clinicaltrials.gov/ct2/ show/NCT02266758.

40. Fagulha I. Diabetes e gravidez. Passado, presente e futuro. Acta Med Port. 2002;15:221-31. 